\title{
Photometric methods for stellar parameter determinations
}

\author{
M S Bessell \\ Research School of Astronomy and Astrophysics, The Australian National University, \\ Mt Stromlo, Cotter Road, Weston, ACT 2611, Australia \\ E-mail: bessell@mso.anu.edu.au
}

Received 17 September 2008

Accepted for publication 19 September 2008

Published 19 December 2008

Online at stacks.iop.org/PhysScr/T133/014019

\begin{abstract}
A star's effective temperature, effective gravity and metallicity is reflected in its energy distribution and line spectrum. Standard photometric systems have been devised to measure these stellar parameters from the stellar fluxes. The most precise of the standard systems can measure magnitude differences (colours) to between 0.1 and $1 \%$. These colours are generally calibrated empirically using stars of known parameters or by synthetic photometry from model atmospheres. This review will highlight the successes and challenges in synthetic photometry calibrations and the prospective improvement in metallicity sensitivity for halo stars with a new sky survey.
\end{abstract}

PACS number: 97.10.Ex

(Some figures in this article are in colour only in the electronic version.)

\section{Introduction}

The stellar journey of the past 40 years has seen revolutionary changes in instrumentation for spectroscopy and photometry. Photoelectric photometry reached its zenith in the 1980s and has now all but vanished, being replaced by CCDs. Individual photometric observations are being replaced by wide-field photometric catalogues. Instrumentation changes usually produced passband changes that have resulted in systematic transformation problems associated with standard photometry.

Spectroscopic detectors moved from photographic plates to image tubes to photon counting devices to CCDs. Echelle spectrographs providing compact full spectral coverage have replaced normal grating spectrographs for high resolution studies. Curves of growth have been replaced by fine analyses and spectrum synthesis; from too little information to a surfeit of information.

Mihalas model atmospheres burst on the world in the mid-1960s, and we have seen the extension and development of these atmospheres by Bengt Gustafsson's group at Uppsala and Bob Kurucz at the Smithsonian Astrophysical Observatory. We owe an immense debt of gratitude to these pioneers of model atmosphere computations for their persistence in making their line opacities more complete and their models more realistic, and in sharing their insight into how to use the results of local thermodynamical equilibrium (LTE) atmosphere analysis to obtain accurate relative abundances, temperatures and gravities.

Throughout this period, detractors have persistently questioned the adoption of LTE in stellar atmospheres. However, in most cases, LTE has provided more reliable interpretations than the hitherto computationally inadequate non-LTE approaches. To paraphrase Winston Churchill, LTE is the worst possible assumption for model atmosphere computations, except for all the others that have been tried. However, that situation is changing.

Over the past 5-10 years, there has been a great increase in computing power and we are now looking toward grids of three-dimensional (3D) and non-LTE model atmospheres. There have been some spectacular successes in the interpretation of the solar spectrum using 3D modeling (Asplund 2005) and the extension to halo giants and dwarfs is impressive (Collet et al 2007). Large corrections have been predicted for abundances based on molecular species and neutral species in very metal-deficient $\mathrm{G}$ and $\mathrm{K}$ stars; however, the treatment of radiative transport in these models is still crude compared with that currently employed in 1D LTE models, and the extremely low temperatures in the outer layers of the 3D halo models should be treated with reserve. Similar 
reserve should be extended to some of the non-LTE analyses until the effects of hydrogen collisions is better understood and modeled.

There are additional challenges associated with modeling the coolest stars as the range of temperatures explored is extended from $3000 \mathrm{~K}$ to below $1000 \mathrm{~K}$, the realm of gas-giant planets. In these atmospheres, the formation of polyatomic molecules and dust has a major influence on the spectrum of the star and the nature of the dust formation and distribution in the atmospheres is still very uncertain.

From the earliest abundance analyses of solar-type dwarfs and $\mathrm{K}$ giants by Holweger et al, it was clear that the most important parameter to determine was the stellar temperature and that the most reliable way to do that was by using photometric colours. The choice of what colour and what temperature calibration was also very important; that is, to choose a colour that showed a large change with temperature and was insensitive to metallicity and a temperature calibration based on fundamental temperatures or the best model atmospheres. For many years, the photometric calibrations of Bell and Gustafsson (1978) provided the basis of many abundance analyses. My involvement with photometric systems was started in order to determine more reliable temperatures for stars covering a wider range of temperatures, luminosities and abundances. A review of standard photometric systems is given in Bessell (2005).

\section{Model atmospheres-derivation of stellar parameters}

Mihalas (1965) atmospheres were the first to show quantitatively how the underlying stellar continua were affected by temperature and gravity. This was illustrated vividly in the figures of Oke (1965). The slope of the Paschen continuum is a sensitive temperature indicator for A, F, G and $\mathrm{K}$ stars. The Balmer jump, or the magnitude difference between the red end of the Balmer continuum and the blue end of the Paschen continuum, is a sensitive temperature indicator for B stars and an effective gravity indicator for F, $\mathrm{G}$ and $\mathrm{K}$ stars. The effective gravity also affects the Paschen continuum in A, F and G stars, the lower the gravity the bluer the slope.

Mihalas also showed that the hydrogen lines were gravity indicators in $\mathrm{O}$ and $\mathrm{B}$ stars but were quite insensitive to gravity in $\mathrm{F}, \mathrm{G}$ and $\mathrm{K}$ stars, making them excellent reddening-free temperature indicators. Barklem (2008) has undertaken a theoretical study of the broadening of hydrogen lines in cool stars, which will enable them to be better used for absolute temperature determinations.

The metal-line blanketed model atmospheres of Kurucz (1979) and Gustafsson et al (1975) provided a great advance in fitting stellar energy distributions. The very influential paper by Gustafsson and Bell (1979) illustrated the promise and challenges of cool stellar model atmospheres, synthetic spectra and theoretical colours, in particular, the importance of molecular lines in cool stars. The most recent blanketed fluxes (ATLAS: Munari et al 2005; MARCS: Gustafsson et al 2008) are excellent matches to observations.

\section{Standard photometric systems}

In figure 1 are shown the passbands of some broad-band and intermediate-band standard photometric systems to indicate the portion of the spectrum the different systems sample. These systems are discussed in more detail in Bessell (2005).

Synthetic photometry traditionally presupposes that a passband exists, which when convolved with a flux spectrum will generate a magnitude identical to the standard magnitude for stars of all temperature, effective gravity and metallicity. The problem is that most standard systems no longer correspond to a natural photometric system but reflect complicated linear and nonlinear transformations of a contemporary natural system. We should therefore expect to use similar conversions for our synthetic photometry.

\subsection{UBV photometry}

The $U B V$ system (Johnson and Morgan 1953) was based around the sensitivity of the $1 \mathrm{P} 21$ phototube. The $U-B$ versus $B-V$ diagram can be used to distinguish dwarf main-sequence stars, reddened OB stars, weak-line (halo) FG stars and evolved $\mathrm{K}$ giants. The $B-V$ colour was normally used to derive temperature, however, for $\mathrm{OB}$ stars the $Q$ method or intrinsic $U-B$ colour is better. The $Q$ method is an excellent technique to correct for interstellar reddening in OB stars. $Q=(U-B)-0.72(B-V)$ and $(B-V)_{0}=$ $-0.009+0.337 Q$. However, the switch to $C C D$ detectors badly impacted $U$ band photometry. Many CCDs, especially the early ones, had very poor UV response and, hence, $U$ band photometry was either not done at all, or the $U$ band was so different from the standard $U$ band that large systematic differences occured.

The fundamental disadvantage of the $U B V$ system is the fact that for FGK stars, $B-V$ is sensitive to temperature, metallicity and effective gravity whereas $U-B$ is sensitive to metallicity and effective gravity.

\section{2. uvby photometry}

Strömgren (1966) also devised the uvby system around the 1P21, but he used narrower passbands designed to better determine the Paschen slope, the Balmer jump and metal-line blanketing in FG stars. This has been a very successful photometric system that is in widespread use. Its power in the study of halo stars can be seen in the work of Schuster et al (2004) where the $c 1$ versus $b-y$ diagram is used to readily distinguish dwarfs, subgiants, horizontal branch stars and sdOB stars.

Unfortunately, the width and position of the $u$ and $v$ bands used by different observers have not been the same as those of the original system, and this has introduced systematic differences in photometry for stars of different metallicities and luminosities. Figure 2 shows the passbands of the following: the original Crawford uvby system: green; the Cousins E-region photometry: red; and Olsen 6-channel photometer: black. Önehag (this conference) illustrates some of the problems associated with synthesizing contemporary $c 1$ and $m 1$ photometry using the original Crawford passbands. 

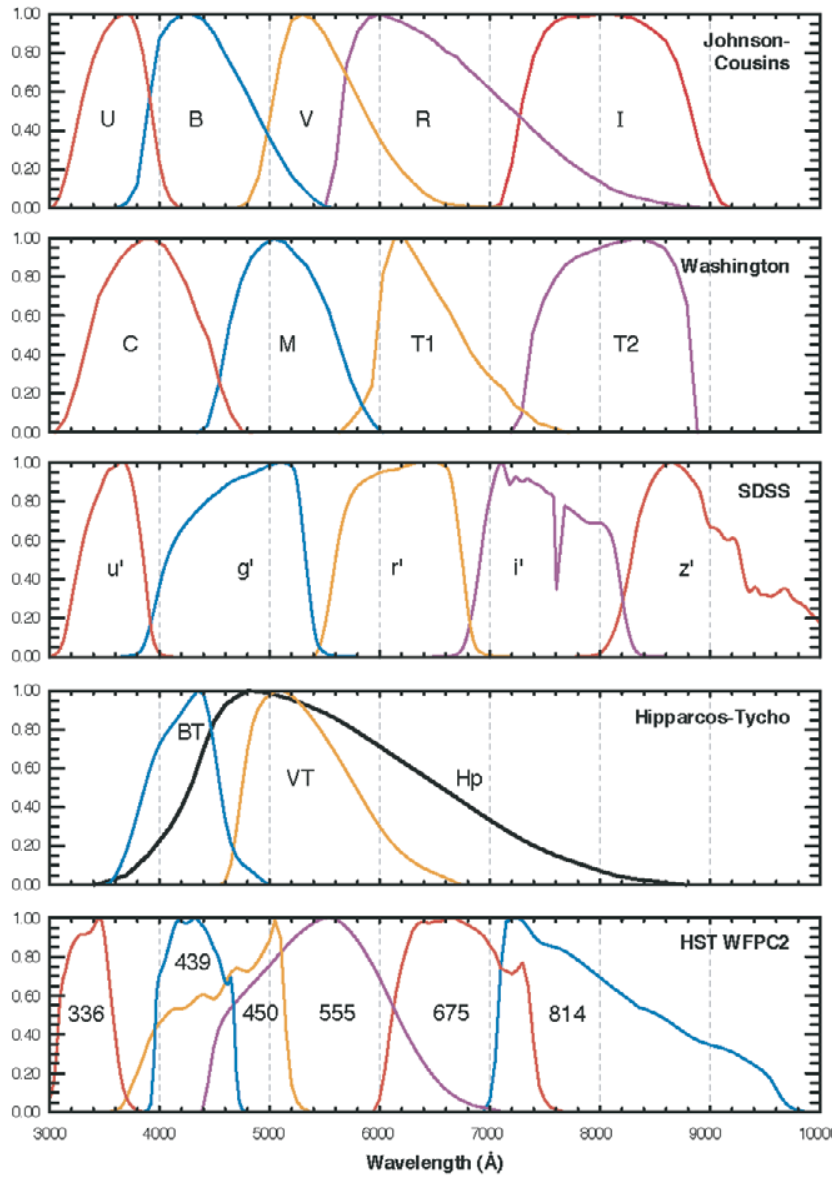
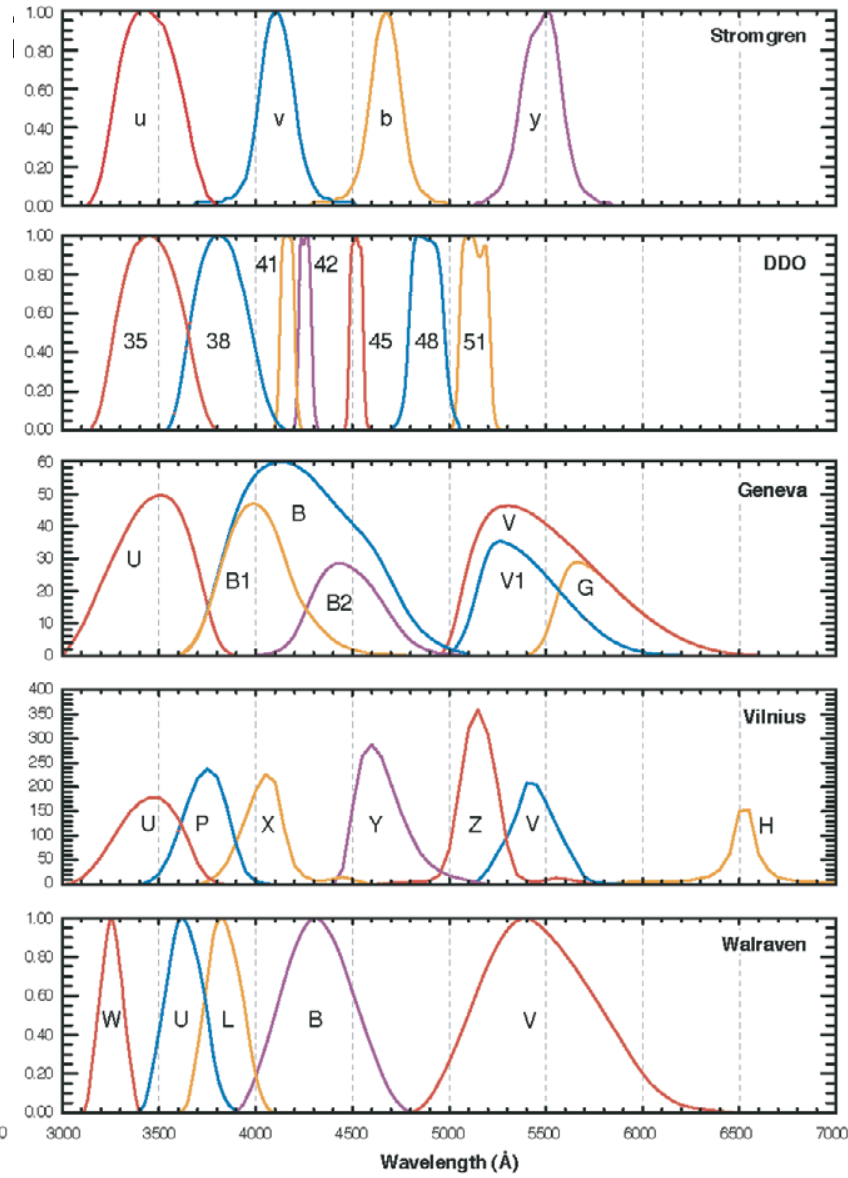

Figure 1. The passbands of some broadband and intermediate-band systems.

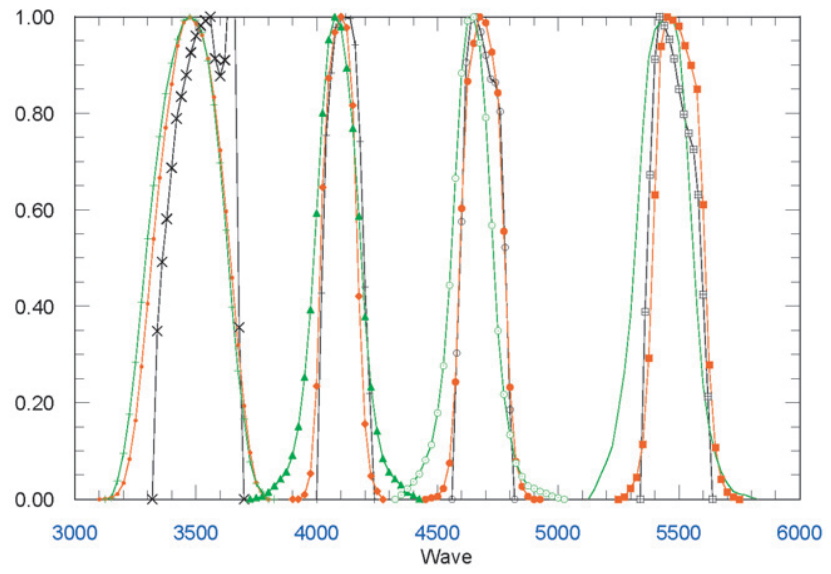

Figure 2. The $u v b y$ passbands of Crawford (green), Cousins (red) and Olsen (black).

However, the photometry of Olsen $(1983,1993)$ has been done mostly with the Danish 6-channel photometers that have virtually identical passbands and that have been matched by the CCD uvby passbands of the Danish telescope used by Grundahl et al (2002) in recent precise globular cluster photometry. It should therefore be possible to better synthesize uvby photometry using the Olsen/Schuster passbands (see Bessell 2005) and transformation equations from Schuster and Nissen (1988).

\subsection{RI and JHKL photometry}

Redder colours that are much less sensitive to metallicity and thus better temperature indicators were provided by different detectors to the blue sensitive 1P21. Johnson (1966) was the first to exploit this with an S-1 based RI system and introduced near-IR JHKL photometry. Cousins (e.g. Kilkenny and Cousins 1995) provided an extremely precise RI extension of the UBV system and showed that photometric precision was not related to the width of the passbands but to the care of the observer. The original red detectors were much less sensitive than the 1P21 and had higher noise; however, with the introduction of the GaAs phototube and now with CCDs the VRI bands have become the most sensitive. With the development of the Mercatel IR arrays, the JHK bands are now within reach for most stars, and colours based on the VRIJHK photometry provide the most accurate temperature determinations for cool stars.

\section{Metallicity sensitivity with wavelength}

The availability of line-blanketed fluxes for a wide range of metallicities enables us to explore the effect of metallicities on fluxes and to understand why some colours and some wavelength regions are better suited for metallicity determinations. For example, figure 3 shows the variation in line blanketing with metallicity for two dwarfs of different temperatures. 

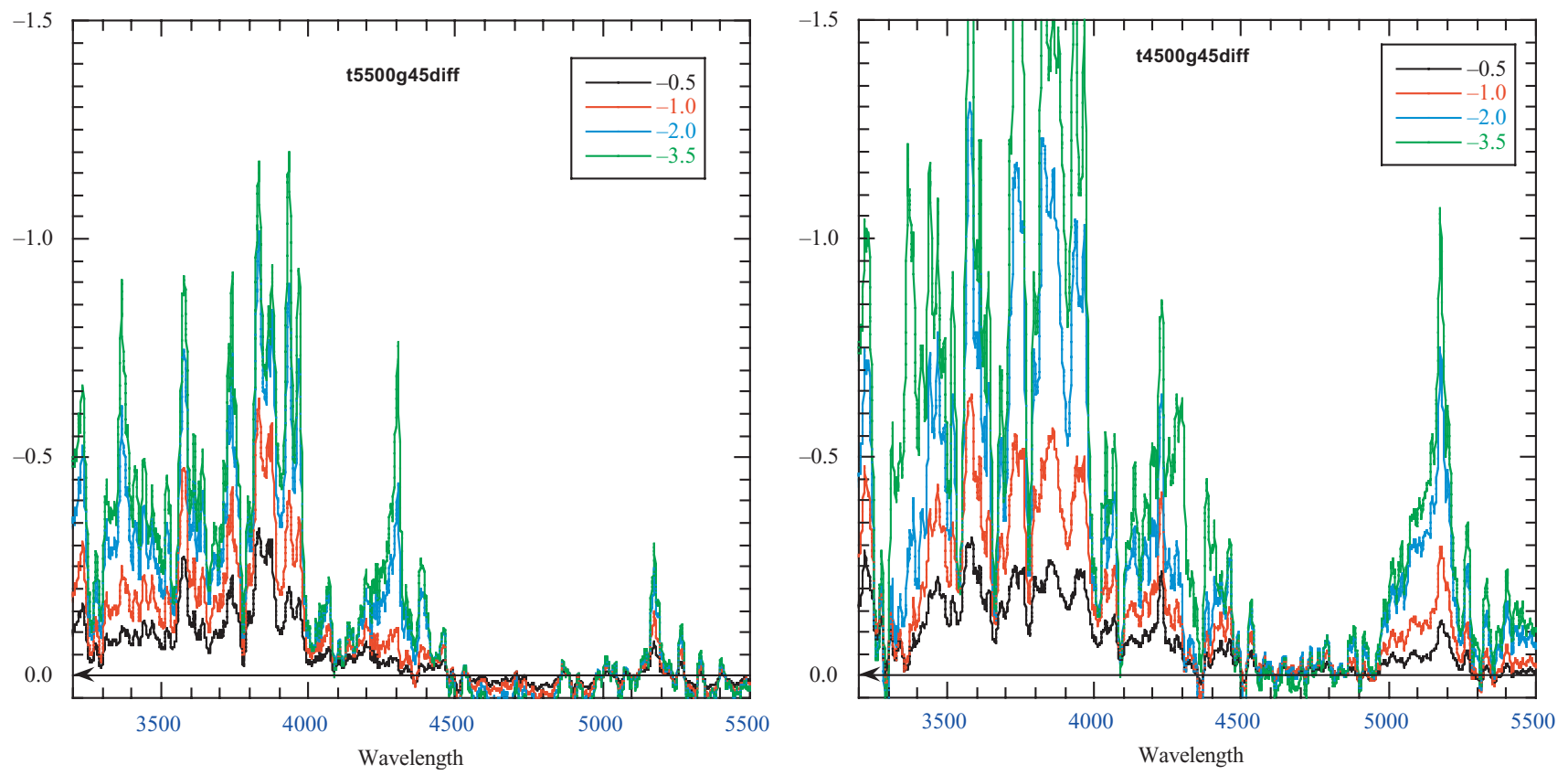

Figure 3. The metal-line blanketing for two different temperature dwarfs.

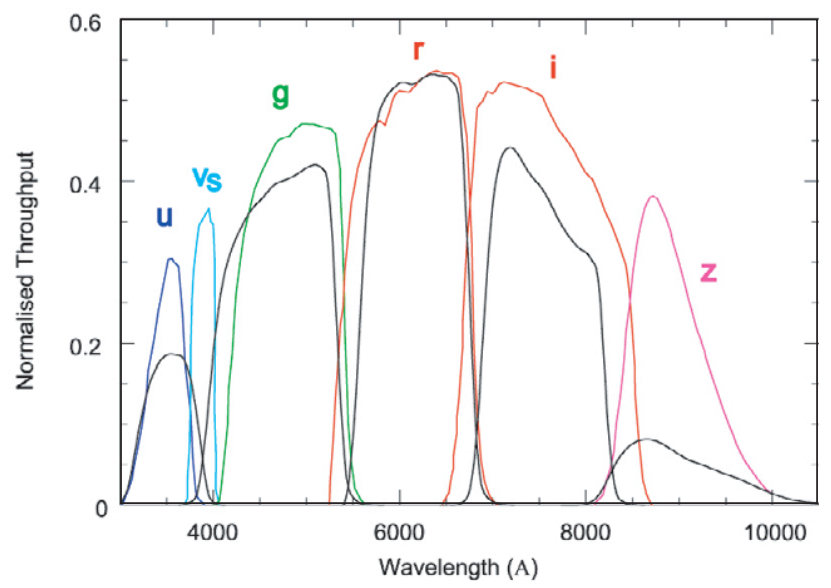

Figure 4. The designed passbands of the SkyMapper Survey. SDSS are in black.

From this, one can see that the Strömgren $b$ band (centered near $4550 \AA$ ) is indeed insensitive to metallicity but that the Strömgren $v$ band centred at $4100 \AA$ is also relatively insensitive to metallicity compared with the bands between 3800 and $4000 \AA$. In order to increase the metallicity sensitivity at warmer temperatures and for the lowest metallicities, it is better to use a band like the DDO38 than the Strömgren $v$ band. This is what the SkyMapper Survey is doing.

\section{The SkyMapper Survey}

Keller et al (2007) are planning an accurate wide-field photometric survey of the southern sky with griz bands similar to the SDSS bands but with a modified $\mathrm{u}$ band like Strömgren $u$ and an additional $v_{s}$ band like the DDO38 band. Figure 4 shows the SkyMapper passbands in comparison with the SDSS passbands. We anticipate achieving limiting magnitudes between 22 and 23 and should be able to measure metallicities to below $[\mathrm{Fe} / \mathrm{H}]=-4$ for halo main-sequence and subgiant stars. The better placed $u$ band will in addition facilitate the determination of the effective gravity of cool stars.

\section{Synthetic spectra and colours}

Over the last 30 years, the major line-blanketed model atmosphere grids were the MARCS and ATLAS grids. The ATLAS grid (Kurucz 1979) and later versions covered from $25000 \mathrm{~K}$ to $4000 \mathrm{~K}$, whereas the MARCS grid (Gustafsson et al 1975) initially concentrated on GK stars and the latest version (Gustafsson et al 2008) ranges from $8000 \mathrm{~K}$ to $3000 \mathrm{~K}$.

\subsection{MARCS models}

Synthetic colours for various systems and temperature calibrations have been provided for the MARCS model fluxes by Bell et al (1976); Bell and Gustafsson (1978): giants; Vandenberg and Bell (1985): dwarfs; Bell and Gustafsson (1989): Te and colours; Trippico and Bell (1991): DDO; Plez et al (1992): M giants; Brett and Plez (1993): M dwarfs; Bell et al (1994): Te, colours; Bessell et al (1998): Te, UBVRIJHKL; Houdashelt et al (2000): Te, colours; Clem et al (2004): uvby.

\subsection{ATLAS models}

Synthetic colours for various systems and temperature calibrations have been provided for the ATLAS model fluxes by Relyea and Kurucz (1978): Te, uvby; Buser and Kurucz (1978): UBV; Kurucz (1979): Te, UBVRI; Lester et al (1986): uvby- $\beta$; Bessell et al (1998): UBVRIJHKL colours; Castelli and Kurucz (2006): H $\beta$; Munari et al (2005): ATLAS9 spectra. 


\section{Temperature calibrations}

Direct radii measurements of stars provide the basic fundamental temperature calibrations; that is, intensity interferometer observations of the brightest A-F stars (Code et al 1976); lunar occultations of KM giants (Ridgway et al 1980) and more recently, Michelson interferometer observations of KM giants and dwarfs (e.g. Berger et al 2006). However the Infrared flux method pioneered by Blackwell (e.g. Blackwell and Lynas-Gray (1994) and Tsuji et al (1996), and more recently by Alonso et al (1999a, 1999b) and Ramirez and Melendez (2005) provides excellent temperatures for stars. Casagrande (e.g. Casagrande et al 2006) is extending this technique to M dwarfs and discusses aspects of this work in these proceedings. In general, there is excellent agreement between the temperature-colour relations of the model atmosphere and those of the IRFM. However, for the halo dwarfs, Ramirez and Melendez suggest temperatures up to $200 \mathrm{~K}$ hotter than the $\mathrm{H} \beta$ temperature scale and the MOITE scale for M dwarfs cooler than $3000 \mathrm{~K}$ (Casagrande et al 2008) is up to $200 \mathrm{~K}$ cooler than that fitted by the new MARCS model fluxes. These differences need to be understood and the correct scales established.

\section{Absolute flux calibrations for standard photometry}

The flux of $\alpha$ Lyrae provides the absolute flux calibration for the UBVRIJHKL system. Azusienis and Straizys (1969) derived passbands and first calibrated the $U B V$ system; recent calibrations and refined passbands for the UBVRIJHKL systems are cited in Bessell (2005). Some photometric systems such as the SDSS system are calibrated directly onto the $\mathrm{AB}$ magnitude system of absolute fluxes as represented by spectrophotometric standards. Rieke et al (2008) have recently revised the calibration of IR fluxes between 1 and $24 \mu$.

\section{Spectrophotometric atlases}

There are two kinds of spectrophotometric atlases. The first one provides spectra for mean spectral types and luminosity classes and is used mainly for population synthesis. However, they can very usefully be used for investigating photometric passbands by synthesizing colours, regressing one colour against another and comparing with empirical colour-colour regressions. Four valuable atlases in this category are Straizys and Sviderskiene (1972): 3000-10000 $\AA$, resolution $50 \AA$; Gunn and Stryker (1983): 3130-10800 $\AA, 10-20 \AA$; Pickles (1998): $1150-25000 \AA, 5 \AA$; and the super-templates of Cohen et al (2003): 1.2-35 $\mu$.

The second kind of atlas provides the spectra of individual stars. These are indispensible for direct comparison with model atmosphere fluxes and for comparing synthetic colours for individual stars with their observed colours. Importantly, by observing spectrophotometric standard stars, observers can calibrate their own relative absolute spectrophotometry. There are two excellent recent atlases in this category: MILES and the HST/STIS Next Generation Spectral Library. The MILES atlas comprises 985 stars spanning a large range in atmospheric parameters. The spectra cover the restricted wavelength range of $3525-7500 \AA$ at a spectral resolution of $2.3 \AA$, insufficient for use as spectrophotometric standards.

The HST/STIS Next Generation Spectral Library is a very ambitious project. It comprises 378 (600 if STIS is resurrected) high $\mathrm{S} / \mathrm{N}$ spectra of Hipparcos stars covering a wide range in effective temperatures and luminosities distributed over four metallicity groups: very low $[\mathrm{Fe} / \mathrm{H}]<1$; low $1.5<[\mathrm{Fe} / \mathrm{H}]<0.5$; near-solar $0.3<[\mathrm{Fe} / \mathrm{H}]<+0.1$; and super-solar $+0.2<[\mathrm{Fe} / \mathrm{H}]$. The wide wavelength range is from $2000-10200 \AA$. Many of the spectra have systematic deviations of a few per cent in flux between 6500 and $10000 \AA$, depending on the star's placement within the STIS slit. This remaining problem is being addressed. These stars are useful for comparison with model atmosphere fluxes and make excellent spectrophotometric standards, especially the lowest metallicity stars. The latest version of the atlas can be found at http://archive.stsci.edu/prepds/stisngsl/index.html.

Bessell (1999) discussed observational strategies and techniques to ensure accurate spectrophotometry is obtained. The STIS weak-line stars supplement or supersede the revised standards presented in that paper.

\section{Balmer Jump measurements}

The effective gravity in FGK stars can be easily determined from the Balmer Jump. This can be readily measured observationally by dividing the observed spectrum by the spectrum of a He white dwarf with temperatures between 8000 and $12000 \mathrm{~K}$ observed with the same setup. This has the effect of linearizing the continuum above and below the Balmer Jump, making extrapolation to the Balmer Jump simple. The model atmosphere fluxes can be divided by a blackbody of a temperature similar to that of the white dwarf. The accuracy of these determinations are sufficient to distinguish between main-sequence stars, turnoff stars, horizontal branch stars, subgiants and giants. More precise gravities can then be assigned using the effective temperature and a theoretical isochrone. More details and examples are given in Bessell (2007).

\section{Summary}

The most recent synthetic spectra of the LTE model atmosphere grids are good matches to observations for stars with temperatures between $30000 \mathrm{~K}$ and $3000 \mathrm{~K}$. Synthetic colours computed from the fluxes are correspondingly good, provided passbands are well established and transformation equations derived. The effective temperature, effective gravity and metallicity are therefore well determined from synthetic photometry and spectrophotometry with these models. More work is still required to model the cooler M and L dwarfs.

Accurate detailed digital spectral atlases are now available and observers should always aim to obtain calibrated spectrophotometry from their spectroscopic observations.

All-sky surveys are already providing precise broadband magnitudes in the optical and infrared regions. The SkyMapper Survey promises good photometry in the u and $\mathrm{v}$ bands as well. 
The long predicted demise of the era of LTE modelling is near. The next few years will see a maturing of $3 \mathrm{D}$ and non-LTE model atmospheres as the remaining computational and physical hurdles are overcome.

\section{Appendix: Discussion}

Q: (Bengt Gustafsson) Do you agree that it would be a great advantage to be able to calibrate the transmission of filters in situ at the telescope?

A: I do. In fact Stubbs et al (2006) are exploring that on the CTIO $4 \mathrm{~m}$ telescope for use with LSST and PanSTARRS.

Q: (Regner Trampedach) What level of accuracy of absolute spectrophotometry can be achieved with STIS using white dwarfs as calibrators?

A: The current STIS data only comprises precise relative-absolute spectrophotometry. The absolute scale will have to be adjusted using synthetic photometry and comparison with catalog V or Hipparcos magnitudes. The STIS relative absolute spectrophotometry still has some systematic deviations of up to $5 \%$ in the red due to the positioning of the star in the STIS slit. Work is underway to correct this and preliminary indications are that this can reduce the uncertainties to better than $2 \%$.

\section{References}

Alonso A, Arribas S and Martinez-Rodger C 1999a Astron. Astrophys. 139353

Alonso A, Arribas S and Martinez-Rodger C 1999b Astron. Astrophys. 140261

Asplund M 2005 Annu. Rev. Astron. Astrophys. 43481

Ažusienis A and Straižys V 1969 Sov. Astron. 13316

Barklem P 2008 in preparation

Bell R A, Eriksson K, Gustafsson B and Nordlund A 1976 Astron. Astrophys. 2337

Bell R A and Gustafsson B 1978 Astron. Astrophys. 34229

Bell R A and Gustafsson B 1989 Mon. Not. R. Acad. Sci. 236653

Bell R A, Paltoglou G and Trippico M J 1994 Mon. Not. R. Acad. Sci. 268771

Berger D H et al 2006 Astrophys. J. $\mathbf{6 4 4} 475$

Bessell M S 1999 Publ. Astron. Soc. Pac. 1111426

Bessell M S 2005 Annu. Rev. Astron. Astrophys. 43293

Bessell M S 2007 Publ. Astron. Soc. Pac. 119605

Bessell M S, Castelli F and Plez B 1998 Astron. Astrophys. 333231

Blackwell D E and Lynas-Gray A E 1994 Astron. Astrophys. 282 899

Brett J M and Plez B 1993 Publ. Astron. Soc. Aust. 10250
Buser R and Kurucz R L 1978 Astron. Astrophys. 70555

Casagrande L, Flynn C and Bessell M 2008 Mon. Not. R. Astron. Soc. 389585

Casagrande L, Portinari L and Flynn C 2006 Mon. Not. R. Acad. Sci. 37313

Castelli F and Kurucz R L 2006 Astron. Astrophys. 454333

Code A D, Davis J, Bless R C and Hanbury Brown R 1976 Astrophys. J. 203417

Cohen M, Wheaton, Wm A and Megeath S T 2003 Astron. J. 126 1090

Collet R, Asplund M and Trampedach R 2007 Astron. Astrophys. 469687

Clem J L, VandenBerg D A, Grundahl F and Bell R A 2004 Astron. J. 1271227

Grundahl F, Stetson P B and Andersen M I 2002 Astron. Astrophys. 395481

Gunn J E and Stryker L L 1983 Astrophys. J. Suppl. 52121

Gustafsson B and Bell R A 1979 Astron. Astrophys. 74313

Gustafsson B, Bell R A, Eriksson K and Nordlund A 1975 Astron. Astrophys. 42407

Gustafsson B, Edvardsson B, Eriksson K, Grae Jorgensen U, Nordlund A and Plez B 2008 in preparation

Houdashelt M L, Bell R A, Sweigert A V and Wing R F 2000 Astron. J. 1191424

Johnson H L and Morgan W W 1953 Astrophys. J. 117313

Johnson H L 1966 Annu. Rev. Astron. Astrophys. 4193

Keller S C et al 2007 Publ. Astron. Soc. Aust. 241

Kilkenny D and Cousins A W J 1995 Astrophys. Space Sci. 230155

Kurucz R L 1979 Astrophys. J. Suppl. 401

Lester J, Gray R O and Kurucz R L 1986 Astrophys. J. Suppl. 61509

Mihalas D 1965 Astrophys. J. Suppl. 929

Munari U, Sordo R, Castelli F and Zwitter T 2005 Astron. Astrophys. $\mathbf{4 4 2} 1127$

Oke J B 1965 Annu. Rev. Astron. Astrophys. 323

Olsen E H 1983 Astron. Astrophys. Suppl. 5455

Olsen E H 1993 Astron. Astrophys. Suppl. 10289

Pickles A J 1998 Publ. Astron. Soc. Pac. 110863

Plez B, Brett J M and Nordlund A 1992 Astron. Astrophys. 256551

Ramirez I and Melendez J 2005 Astrophys. J. 626446

Relyea L J and Kurucz R L 1978 Astrophys. J. Suppl. 3745

Ridgway S T, Joyce R R, White N M and Wing R F 1980 Astrophys. J. 235126

Rieke G H et al 2008 Astron. J. 1352245

Schuster W J and Nissen P E 1988 Astron. Astrophys. Suppl. 73225

Schuster W J, Beers T C, Michel R, Nissen P E and García G 2004 Astron. Astrophys. 422527

Straizys V and Sviderskiene Z 1972 Vilnius. Astron. Obs. Bull. 353

Strömgren B 1966 Annu. Rev. Astron. Astrophys. 4433

Stubbs C W et al 2006 arXiv:astro-ph/0609260vl

Tsuji T, Ohnaka K and Aoki W 1996 Astron. Astrophys. 305 L1

Tripicco M J and Bell R A 1991 Astron. J. 102744

VandenBerg D A and Bell R A 1985 Astrophys. J. Suppl. 58561 